\title{
Avaliação de argilas bentoníticas policatiônicas do estado da Paraíba com aditivos para aplicação em perfuração de poços de petróleo e tintas à base de água
}

\section{(Evaluation of polycationic bentonite clays from Paraiba State with additives for application in oil drilling and water-based paints)}

\author{
B.M.A. Brito, J.M.Cartaxo, N. F. C. Nascimento, H. C. Ferreira, G. A. Neves, R. R. Menezes \\ UFCG - CCT - UAEMa, R. Aprígio Veloso 882, Campina Grande, PB, 58429-900 \\ bruninha.michele@hotmail.com,julianamelo25@gmail.com,neyberfadiocn@gmail.com,heber.ferreira@ufcg. \\ edu.br,gelmires.neves@ufcg.edu.br,romualdomenezes@gmail.com
}

\begin{abstract}
Resumo
Com o surgimento das novas ocorrências de bentonitas no estado da Paraíba, há necessidade da otimização de sua reologia de forma a adequá-las às exigências da indústria nacional, visando seu uso em fluidos de perfuração e tintas à base de água. Foram utilizadas três argilas (Chocolate Campos Novos, Chocolate Corredor e Chocobofe Primavera) com o objetivo de avaliar as propriedades tecnológicas para uso como agente tixotrópico na formulação de fluidos de perfuração e tintas à base de água; esta pesquisa trata da reologia de dispersões de argilas aditivadas com $\mathrm{MgO}$ de forma conjunta e isolada com o $\mathrm{Na}_{2} \mathrm{CO}_{3}$. $\mathrm{O}$ comportamento reológico foi estudado em viscosímetro em dispersões de $4,86 \%$ e $6,00 \%$ (em massa) e com aditivos variando de $2 \%$ a $3 \%$ (em massa) na forma simples e combinada. Os resultados obtidos foram comparados com as especificações da Petrobras, API e ISO. As amostras estudadas apresentaram características típicas das bentonitas oriundas do estado da Paraíba, apresentando os argilominerais característicos esmectíticos, cauliníticos e acessórios; as análises reológicas evidenciaram que as argilas em estudo utilizando o $\mathrm{Na}_{2} \mathrm{CO}_{3}$ exibiram resultados promissores para aplicação tecnológica em fluidos de perfuração de poços e que a aditivação das amostras com $\mathrm{Na}_{2} \mathrm{CO}_{3}$ em conjunto com o $\mathrm{MgO}$ exibiram resultados promissores para aplicação tecnológica em tintas à base de água.

Palavras-chave: argilas bentonitas, propriedades reológicas, fluidos de perfuração, tintas à base de água.
\end{abstract}

Abstract

With the new occurrences of bentonite clays in Paraiba State, there is need for optimizing their rheology in order to make them to fit the requirements of the Brazilian industry, aiming its use in drilling fluids and water based paints. Three clays were used (Chocolate Campos Novos, Chocolate Corredor and Chocobofe Primavera) in order to assess the technological properties for use as thixotropic agent in the formulation of drilling fluids and water based inks; this research deals with the rheology of dispersions of doped clay with $\mathrm{MgO}$ and/or $\mathrm{Na}_{2} \mathrm{CO}_{3}$. The rheological behavior was studied with a viscometer with dispersions of $4.86 \%$ and $6.00 \%(w / w)$ and with additives ranging from $2 \%$ to $3 \%(w / w)$ in simple and combined form. The results obtained were compared with the Petrobras, ISO and API specifications. The studied samples showed typical characteristics of bentonite originating from Paraiba State, with the characteristic smectite and kaolinitic clay minerals, and accessories; the rheological analysis showed that the clays studied using $\mathrm{Na}_{2} \mathrm{CO}_{3}$ showed promising results for technological application in well drilling fluids, and the samples with both additives $\mathrm{Na}_{2} \mathrm{CO}_{3}$ and $\mathrm{MgO}$ exhibited promising results for technological application in water based paints.

Keywords: bentonite clays, rheological properties, drilling fluids, water based paints.

\section{INTRODUÇÃO}

As argilas bentoníticas são argilas formadas pela devitrificação e subsequente alteração química de tufos ou cinzas vulcânicas, constituídas essencialmente pelo argilomineral montmorilonita pertencente ao grupo das esmectitas. Essas argilas são constituídas por lamelas formadas por uma folha octaédrica de alumina $\left(\mathrm{Al}_{2} \mathrm{O}_{3}\right)$ entre duas folhas tetraédricas de sílica $\left(\mathrm{SiO}_{2}\right)$. As bentonitas apresentam amplo uso industrial, sendo utilizadas na pelotização de minério de ferro, ligantes de areias em moldes para fundição, na clarificação de óleos, impermeabilizantes de barragens, em fluidos ou lamas de perfuração, etc. [13]. A sua composição mineralógica e parâmetros físicoquímicos são cruciais para determinar seu desempenho nas mais variadas aplicações industriais. As propriedades importantes das esmectitas incluem elevadas capacidade de troca de cátions (CTC), área específica e capacidade de absorção e inchamento, assim como altas viscosidades e tixotropia, o que permitem uma vasta gama de aplicações [4]. Os maiores depósitos de argilas bentoníticas do mundo estão concentrados nos EUA, sendo que o Brasil se destaca entre os dez principais produtores mundiais, explorando depósitos relativamente pequenos, sendo os 
mais importantes localizados no Nordeste, no município de Boa Vista - PB, representando cerca de $90 \%$ da produção nacional [5]. De acordo com o cátion de compensação presente na argila, as bentonitas podem ser monocationicas ou policatiônicas. Bentonitas policatiônicas, quando transformadas em sódicas pelo tratamento com carbonato de sódio, incham na presença de água, aumentando várias vezes seu volume inicial, sendo utilizadas na preparação de fluidos de perfuração e tintas à base de água devido às propriedades reológicas que apresentam [6]. Os fluidos de perfuração são sistemas formados por um meio dispersante e fase dispersa, sendo esta composta por componentes inorgânicos e orgânicos. Sendo assim, três são as frações do fluido: líquida, coloidal e inerte. A primeira relativa ao meio dispersante; a segunda, associada aos compostos argilosos que aumentam a viscosidade do meio (argilas esmectíticas/bentonitas), e a inerte que é típica de detritos oriundos das rochas perfuradas, que quando menores que $2 \mu \mathrm{m}$ podem ser incorporadas à fração coloidal. O sucesso da perfuração de um poço depende, dentre outros fatores, das características físico-químicas do fluido de perfuração utilizado na operação [7]. A composição do fluido é escolhida de modo que se tenha as propriedades ideais, como viscosidades adequadas, consistência de gel, controle de filtrado, reboco, inibição de argilas hidratáveis da formação geológica e coeficiente de lubricidade.

No âmbito da perfuração de poços, as exigências ambientais estão cada vez mais severas, fazendo com que se estude e busquem novos desenvolvimentos para os fluidos a base de água. Os fluidos a base de água estão em crescente utilização, visto que fluidos a base de óleo tradicionais com óleo diesel, embora apresentem desempenho superior e sejam mais estáveis a altas pressões e temperaturas, são altamente tóxicos e de custo elevado [8-10]. A normatização para a determinação e controle dos fluidos de perfuração é feita, dentre outras, pela Petrobras [11], American Petroleum Institute (API) [12] e International Organization for Standardization (ISO) [13]. A norma da Petrobras [11] recomenda para uma dispersão de $4,86 \%$ (em massa) uma viscosidade aparente (VA) de $15 \mathrm{mPa} . \mathrm{s}$ a $600 \mathrm{rpm}$, viscosidade plástica (VP) de $4 \mathrm{mPa} . \mathrm{s}$ (600 e $300 \mathrm{rpm}$ ) e volume de filtrado (VF) de $18 \mathrm{~mL}$. As normas da API [12] e ISO [13] recomendam para uma dispersão de 6,00\% (em massa) uma VA de $15 \mathrm{mPa}$.s a $600 \mathrm{rpm}$ e VF de $15 \mathrm{~mL}$. A normatização de esmectitas aditivadas para uso como agentes espessantes de tintas à base de água são as mesmas da API [12] e ISO [13] destinadas a fluidos de perfuração à base de água de poços de petróleo. Os conceitos e a determinação de viscosidade aparente (VA), viscosidade plástica (VP) e volume de filtrado (VF) estão também descritos em [14, 15]. As dispersões de argilas bentoníticas apresentam um estado intermediário entre floculadas e defloculadas, sendo que as mais defloculadas apresentam o fenômeno de tixotropia, enquanto que as mais floculadas apresentam o fenômeno de reopexia [16]. O comportamento tixotrópico resulta do tratamento efetuado com agentes defloculantes, tais como $\mathrm{Na}_{2} \mathrm{CO}_{3}, \mathrm{NaOH}$, etc., enquanto que o comportamento reopético resulta de aditivações com agentes floculantes, tais como $\mathrm{Ca}(\mathrm{OH})_{2}$ e $\mathrm{Mg}(\mathrm{OH})_{2}$. Comportamentos intermediários entre os dois estágios acima mencionados podem ser obtidos através da aditivação conjunta de agentes floculantes e defloculantes em proporção adequada de forma a atingir uma reologia especificada para um determinado uso industrial. Aditivações inorgânicas se sobressaem ao serem comparadas às orgânicas quanto à relação custo-benefício e, também, não degradam, muito embora as aditivações orgânicas sejam mais eficientes como espessantes de fluidos de perfuração e tintas à base de água [17]. Assim sendo, o tratamento de argilas esmectíticas com sódio aumenta o número de partículas finas hexagonais lamelares, enquanto íons $\mathrm{Ca}^{2+}$ formam estruturas em bandas e íons $\mathrm{Mg}^{2+}$ estruturas em rede ou tridimensionais $[1,18]$. As dispersões com grau de floculação intermediário são tipicamente usadas em fluidos de perfuração, enquanto que as dispersões floculadas, denominadas de floculadas-gel, formadas por um macrofloco tridimensional (castelo de cartas), são usadas como agentes espessantes de tintas. As aditivações secundárias de bentonitas sódicas com $\mathrm{MgO}$ sofrem influência marcante do $\mathrm{pH}$ resultando na formação de $\mathrm{Mg}^{2+}, \mathrm{MgOH}^{+}$e $\mathrm{Mg}(\mathrm{OH})_{2}$. pHs acima de 9,5 transformam $\mathrm{o} \mathrm{Mg}^{2+}$ em $\mathrm{MgOH}^{+}$e $\mathrm{Mg}(\mathrm{OH})_{2}$, dependendo da concentração de $\mathrm{MgO}$ na dispersão [19]. A formação de $\mathrm{MgOH}^{+}$resulta em altas viscosidades pela estrutura do gel formado. Quando $\mathrm{o} \mathrm{Mg}(\mathrm{OH})_{2}$ é formado em $\mathrm{pHs}$ mais altos, é precipitado juntamente com as partículas do argilomineral havendo heterocoagulação, destruindo a estrutura de castelo de cartas existente, resultando na separação de fases do sistema e com redução da viscosidade aparente (VA).

Modernamente as tintas à base de água são recomendadas em substituição às tintas à base de óleo em virtude de conduzirem a problemas ambientais de importância secundária e também em virtude de problemas econômicos resultantes do custo relativamente baixo dos materiais argilosos [18]. Também foi observado que o uso do $\mathrm{MgO}$ proporciona um maior efeito reopético às suspensões bentoníticas à base de água destinadas à indústria de tintas e correlatas [18]. Deve ser salientado que não existem duas argilas iguais [1]; além deste fato, as argilas são produtos naturais que sofrem um processo de beneficiamento (desaglomeração, aditivação e secagem) contendo também minerais acessórios, tais como mica, quartzo, feldspato e matéria orgânica. Desta forma, o comportamento destes produtos é extremamente complexo e frequentemente os modelos teóricos não se aplicam de forma integral aos resultados obtidos com diferentes bentonitas. As argilas de Boa Vista-PB tradicionalmente apresentam viscosidades plásticas muito reduzidas da ordem de $4 \mathrm{mPa} . \mathrm{s}$, o que se constitui um ponto crítico, pois este é o limite inferior de viscosidade plástica da norma da Petrobras [11]. Acreditase que a norma foi alterada de $8 \mathrm{mPa} . s$ para $4 \mathrm{mPa}$.s em virtude das limitações nesta propriedade. A crescente preocupação para manter a adequação futura dos recursos necessários à indústria de petróleo e gás impulsiona a mineração para explorar novas áreas, de forma a contribuir no desafio que surge causado pelas mudanças na profundidade operacional, o aumento do comprimento da perfuração horizontal para 
melhorar a produção, dentre outros fatores [20]. No contexto atual de esgotamento das jazidas de Boa Vista-PB e no surgimento/estudo das novas ocorrências nos municípios de Sossego-PB, Cubati-PB e Pedra Lavrada-PB, há uma marcante necessidade da otimização da reologia destas argilas de forma a adequá-las às exigências da indústria nacional, evitando o oneroso processo de importação. Desta forma, a presente pesquisa teve como objetivo tratar as dispersões destas argilas com $\mathrm{Na}_{2} \mathrm{CO}_{3}$ de forma conjunta e isolada com $\mathrm{MgO}$, seguindo as orientações de [18, 19], visando seu uso em fluidos de perfuração à base de água e tintas à base de água.

\section{MATERIAIS E MÉTODOS}

Foram estudadas três amostras de argilas esmectitícas fornecidas pela Bentonit União Nordeste (BUN, Campina Grande, PB) denominadas de: Chocolate Campos Novos (CH CN, Cubati - PB); Chocolate Corredor (CH CO, Boa Vista - PB); Chocobofe Primavera (CB PR, Boa Vista $\mathrm{PB})$. Os aditivos usados para tratamento das bentonitas foram o $\mathrm{Na}_{2} \mathrm{CO}_{3}$ sólido (Jand Química) e $\mathrm{MgO}$ obtido a partir do $\mathrm{Mg}(\mathrm{OH})_{2}$ (Labsynth) calcinado a $800{ }^{\circ} \mathrm{C}$ por $2 \mathrm{~h}$. A caracterização física, química e mineralógica das amostras foi realizada pelos seguintes métodos: análise granulométrica por difração de laser (AG, Cilas 1064) em modo úmido; análise química por espectroscopia de fluorescência de raios X (EDX, Shimadzu720); difração de raios X (DRX, Shimadzu XRD6000) com radiação $\mathrm{K} \alpha$ do $\mathrm{Cu}(40 \mathrm{kV} / 30 \mathrm{~mA})$ e velocidade do goniômetro de $2 \%$ min e passo de $0,02^{\circ}$, com faixa de $2^{\circ}$ a $60^{\circ}$. As distâncias interplanares foram calculadas através da Lei de Bragg e foram realizadas pelo software do equipamento. Para a confirmação da presença dos argilominerais do grupo da esmectita, utilizou-se o etileno glicol resultando em um deslocamento do pico característico para 17,0 $\AA$. Nos difratogramas de raios $\mathrm{X}$, as intensidades foram indicadas em contagens por segundo (cps). As análises termogravimétricas (TG) e análises térmicas diferenciais (ATD) foram realizadas a partir de um sistema de análises térmicas (BPEngenharia RB3000 ), com taxa de aquecimento de $12,5^{\circ} \mathrm{C} / \mathrm{min}$, temperatura máxima para ambos de $1000{ }^{\circ} \mathrm{C}$ e o padrão utilizado na ATD foi o óxido de alumínio $\left(\mathrm{Al}_{2} \mathrm{O}_{3}\right)$ calcinado.

As VA e VP foram medidas por meio de viscosímetro (Fann 35A), conforme normas Petrobras [11], API [12] e ISO 13500 [13], segundo as Equações A e B, em cP: A e B, em cP:

$$
\mathrm{VA}=300 \frac{\theta}{\mathrm{N}}
$$

onde, VA é a viscosidade aparente dada em mPa.s, $\mathrm{N}$ é a velocidade de rotação em $600 \mathrm{rpm}$ do viscosímetro Fann e $\theta$ é a deflexão medida no viscosímetro a esta velocidade.

$$
\mathrm{VP}=300\left(\frac{\theta_{2}-\theta_{1}}{\mathrm{~N}_{2}-\mathrm{N}_{1}}\right)
$$

onde, VP é a viscosidade plástica dada em mPa.s, $\theta_{2}$ e $\mathrm{N}_{2}$ com leitura a $600 \mathrm{rpm}$ e $\theta_{1}$ e $\mathrm{N}_{1}$ com leitura a $300 \mathrm{rpm}$. O volume de filtrado (VF) foi determinado através de ensaio realizado em filtro prensa, no qual foi inserida a dispersão e recolhido o filtrado durante o período de $30 \mathrm{~min}$ após a aplicação de uma pressão de $690 \pm 35 \mathrm{kPa}(100 \pm 5$ psi). Para VA, VP e VF, foram efetuadas duas determinações para cálculo do valor médio. Quando a média diferia mais de $\pm 3 \%$ dos valores determinados, um terceiro ensaio foi efetuado, procurando-se satisfazer a variação mencionada. Caso não ocorresse, novos ensaios foram realizados. As bentonitas foram aditivadas de forma individual e combinada, conforme metodologia descrita em [19]. As dispersões foram homogeneizadas por agitador Marconi 500 W com aproximadamente $15000 \mathrm{rpm}$. O comportamento reológico foi estudado em viscosímetro em dispersões de $4,86 \%$ e $6,00 \%$ (em massa) e os aditivos variando $2 \%$ e $3 \%$ (em massa) na forma simples e combinada.

\section{RESULTADOS E DISCUSSÃO}

Na Fig. 1 estão ilustradas as curvas de distribuição de tamanho de partículas das amostras estudadas. As amostras apresentaram comportamento próximo de monomodal, nas quais foi possível observar percentuais variando entre $26 \%$ e $41 \%$ de partículas com diâmetro inferior a $2 \mu \mathrm{m}$. A amostra $\mathrm{CH}$ CN apresentou diâmetro a $50 \%$ em volume cumulativo (D50) de 4,66 $\mu \mathrm{m}$ e diâmetro médio (DM) de $6,78 \mu \mathrm{m}$. A amostra CH CO apresentou D50 de 2,68 $\mu \mathrm{m}$ e DM de 3,84 $\mu \mathrm{m}$. A amostra CB PR apresentou D50 de $3,53 \mu \mathrm{m}$ e DM de 4,51 $\mu \mathrm{m}$. Estes resultados são similares aos descritos por $[3,21,22]$.

Na Tabela I estão contidos os valores das composições químicas das amostras estudadas. Pode-se observar que as amostras analisadas apresentaram elevados teores de óxido de silício variando entre $41 \%$ e $58 \%$ que se referem tanto ao óxido presente na camada tetraédrica do argilomineral como também a sílica livre, ou seja, mineral acessório. Os valores de $\mathrm{Al}_{2} \mathrm{O}_{3}$ evidenciam pequenas variações $(16,05 \%, 16,29 \%$ e $16,40 \%)$ e definem a alumina presente na camada octaédrica e minerais acessórios. Observou-se também que as amostras em estudo apresentaram teores elevados de óxido de ferro, sendo a amostra Chocolate Corredor o mais elevado (9,47\%), característico dos argilominerais do grupo das esmectitas [1]. Estes valores estão associados às substituições isomórficas na camada octaédrica de bentonitas e com minerais acessórios e cátions trocáveis nas camadas basais de argilominerais esmectíticos e cauliníticos. A bentonita Chocolate Campos Novos apresenta teor significativo de $\mathrm{CaO}$ : o cálcio é típico também de substituiçõos na camada octaédrica de esmectitas e constituinte de acessórios, na forma de calcita ou dolomita. Nota-se que as amostras apresentaram perda ao fogo entre $11,6 \%$ e 19,0\%, o que está relacionado à perda de águas coordenadas e adsorvidas, hidroxilas dos argilominerais, carbonatos, matéria orgânica e outros. Comparando os valores determinados com as argilas do município de Cubati, PB [20], e Sossego, PB [3], verifica-se que as amostras em estudo possuem valores similares às argilas bentoníticas desses municípios. 

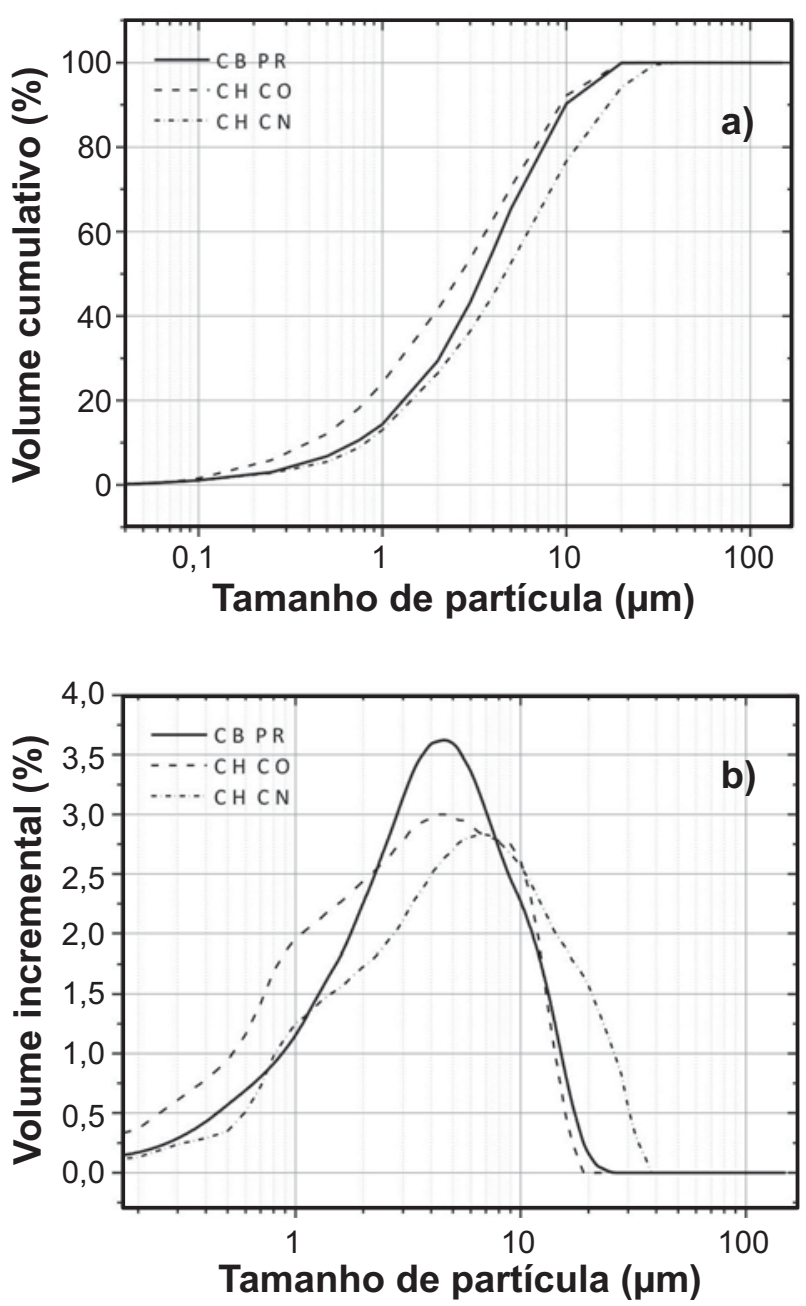

Figura 1: Curvas de distribuição de tamanho de partículas das amostras estudadas: $\mathrm{CH}$ CN, $\mathrm{CH}$ CO e CB PR.

[Figure 1: Particle size distribution curves of analyzed samples: $\mathrm{CH} \mathrm{CN}, \mathrm{CH} \mathrm{CO}$ and $\mathrm{CB}$ PR.]

Na Fig. 2 estão ilustradas as curvas de análises termogravimétricas e térmicas diferenciais das argilas estudadas. Observando as curvas de DTA das amostras em estudo (Fig. 2a), verificam-se as seguintes transformações térmicas: grande pico endotérmico em aproximadamente $106{ }^{\circ} \mathrm{C}$, caracterizando a presença de água livre e adsorvida; pico endotérmico em torno de $499{ }^{\circ} \mathrm{C}$ caracterizando a presença de hidroxilas da folha octaédrica; para a amostra CH CN, observa-se ainda um pico endotérmico com máximo
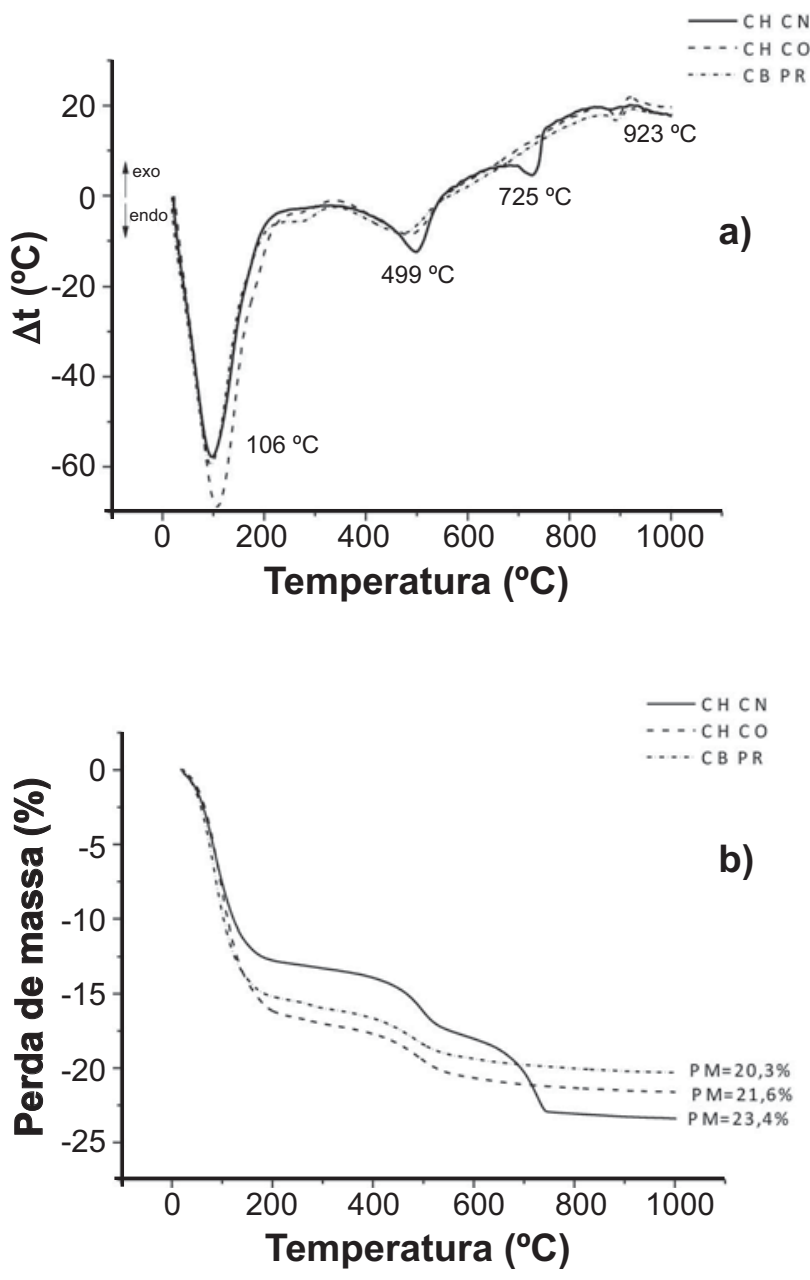

Figura 2: Curvas de análise térmica diferencial (a) e termogravimétrica (b) das argilas estudadas: $\mathrm{CH} \mathrm{CN}, \mathrm{CH}$ $\mathrm{CO}$ e CB PR.

[Figure 2: Curves of differential thermal analysis (a) and thermogravimetry (b) of clays: $\mathrm{CH} C \mathrm{~N}, \mathrm{CH} C \mathrm{O}$ and $\mathrm{CB} P \mathrm{PR}$.]

em $725^{\circ} \mathrm{C}$ caracterizando a presença de carbonato de cálcio e sua decomposição, de acordo com a composição química (Tabela I); e por último a amostra CB PR apresentou um pico exotérmico com máximo em $923{ }^{\circ} \mathrm{C}$, característica da formação da mulita com liberação do quartzo alfa ou beta. Em relação às curvas de TG das amostras em estudo (Fig. 2b), observa-se que a perda total de massa variou entre $20 \%$ e $23 \%$, correspondente às perdas de água, de matéria orgânica, hidroxilas e carbonatos. Comparando os resultados dos

Tabela I - Composição química das argilas estudadas: CH CN, CH CO e CB PR.

[Table I - Chemical composition of clays: $\mathrm{CH} C \mathrm{~N}, \mathrm{CH} \mathrm{CO}$ and $\mathrm{CB}$ PR.]

\begin{tabular}{ccccccccc}
\hline Amostra & $\begin{array}{c}\mathrm{SiO}_{2} \\
(\%)\end{array}$ & $\begin{array}{c}\mathrm{Al}_{2} \mathrm{O}_{3} \\
(\%)\end{array}$ & $\begin{array}{c}\mathrm{MgO} \\
(\%)\end{array}$ & $\begin{array}{c}\mathrm{Fe}_{2} \mathrm{O}_{3} \\
(\%)\end{array}$ & $\begin{array}{c}\mathrm{CaO} \\
(\%)\end{array}$ & $\begin{array}{c}\mathrm{K}_{2} \mathrm{O} \\
(\%)\end{array}$ & $\begin{array}{c}\text { Outros óxidos } \\
(\%)\end{array}$ & $\begin{array}{c}\mathrm{PR} \\
(\%)\end{array}$ \\
\hline CH CN & 41,65 & 16,40 & 3,55 & 8,58 & 8,41 & 0,99 & 1,40 & 19,00 \\
CH CO & 53,80 & 16,29 & 2,38 & 9,47 & 1,11 & 0,41 & 1,54 & 15,00 \\
CB PR & 58,02 & 16,05 & 2,32 & 9,08 & 1,12 & 0,36 & 1,50 & 11,60 \\
\hline
\end{tabular}

PR - Perda ao rubro. 
termogramas com os valores encontrados para as argilas de Pedra Lavrada, PB [22], bem como para as argilas de Cubati - PB [21], e paras as argilas de Sossego - PB [3], observa-se que são similares, evidenciando um comportamento típico das argilas bentoníticas.

Na Fig. 3 estão ilustrados os difratogramas das amostras estudadas. Analisando-se o difratograma da amostra $\mathrm{CH}$ $\mathrm{CN}$, observaram-se presenças de: argilomineral caulinítico, caracterizado pelas distâncias interplanares basais de 7,2, 4,44 e 3,57 ̊̊; quartzo, caracterizado pelas distâncias interplanares de 4,24, 3,34, 2,46,2,28 e 1,9 $\AA$; argilomineral esmectítico nas distâncias interplanares de 15,85 e 3,04 $\AA$, comprovado pelo uso do etileno glicol que aumentou a distância interplanar de 15,85 ̊̊ para 17,0 Å; e calcita com distâncias interplanares de 3,24 e 2,88 $\AA$. Ao analisar o difratograma da amostra $\mathrm{CH} \mathrm{CO}$, observaram-se presenças de: quartzo, caracterizado pelas distâncias interplanares de 4,21, 3,32, 2,12 e 1,8 ̊; e argilomineral esmectítico nas distâncias interplanares de 15,12, 4,41 e $3 \AA$, comprovado pelo uso do etileno glicol que aumentou a distância interplanar de $15,12 \AA$ para $17,0 \AA$. Analisando-se o difratograma da amostra CB PR, observaram-se presenças de: feldspato, caracterizado pelas distâncias interplanares basais de 3,46 e 3,15 ̊; quartzo, caracterizado pelas distâncias interplanares de 4,24, 3,34, 2,45 e 1,97 A; e argilomineral esmectítico nas distâncias $15,49,4,44$ e 2,55 $\AA$, comprovado pelo uso do etileno glicol que aumentou a distância interplanar de $15,49 \AA$ para 17,0 ̊. A partir das análises dos difratogramas, constatase que todas as bentonitas têm argilominerais esmectíticos e cauliníticos, semelhantes às argilas esmectíticas da região de Boa Vista [23] e vizinhanças, como de Pedra Lavrada PB [22], Cubati - PB [21] e Sossego - PB [3]. Os minerais acessórios presentes nas argilas são o quartzo, feldspato e calcita, sendo o último encontrado na bentonita $\mathrm{CH} C \mathrm{CN}$, cuja análise por EDX apontou elevado teor de $\mathrm{CaO}$ na sua composição química. Nota-se ainda no difratograma da amostra $\mathrm{CH} \mathrm{CN}$ uma menor quantidade de argilominerais em todas as análises, o que pode conduzir a uma qualidade inferior na reologia das suspensões.

As dispersões estudadas foram preparadas com 4,86\% em massa para atender as especificações das normas da Petrobras [11] e 6,00\% em massa para atender as especificações das normas da API [12] e ISO [13]. Nas Tabelas II e III estão contidos os resultados das propriedades reológicas obtidas a partir das amostras dispersas a 4,86\% (em massa) e aditivadas isoladamente. Na Tabela II estão contidos os resultados de VA, VP e VF das amostras aditivadas com $\mathrm{Na}_{2} \mathrm{CO}_{3}$ (2\% e $3 \%$ em massa). Observa-se que as dispersões apresentaram VA variando de 3,00 a 13,50 mPa.s, VP variando de 1,50 a 2,00 mPa.s e VF variando de 30,00 até 39,00 $\mathrm{mL}$ para a amostra CH CN. Observa-se VA variando entre 9,75 a 19,00 $\mathrm{mPa} . \mathrm{s}$, VP variando entre 4,00 a 5,00 mPa.s e VF variando entre 17,20 até $20,00 \mathrm{~mL}$, para a amostra $\mathrm{CH} \mathrm{CO}$. Observa-se também VA variando entre 7,00 a 7,75 mPa.s, VP variando entre 4,00 a 4,50 mPa.s e VF variando entre 21,00 até $23,00 \mathrm{~mL}$ para a amostra CB PR. Apenas a amostra $\mathrm{CH} \mathrm{CO}$ aditivada com $3 \%$ em massa de
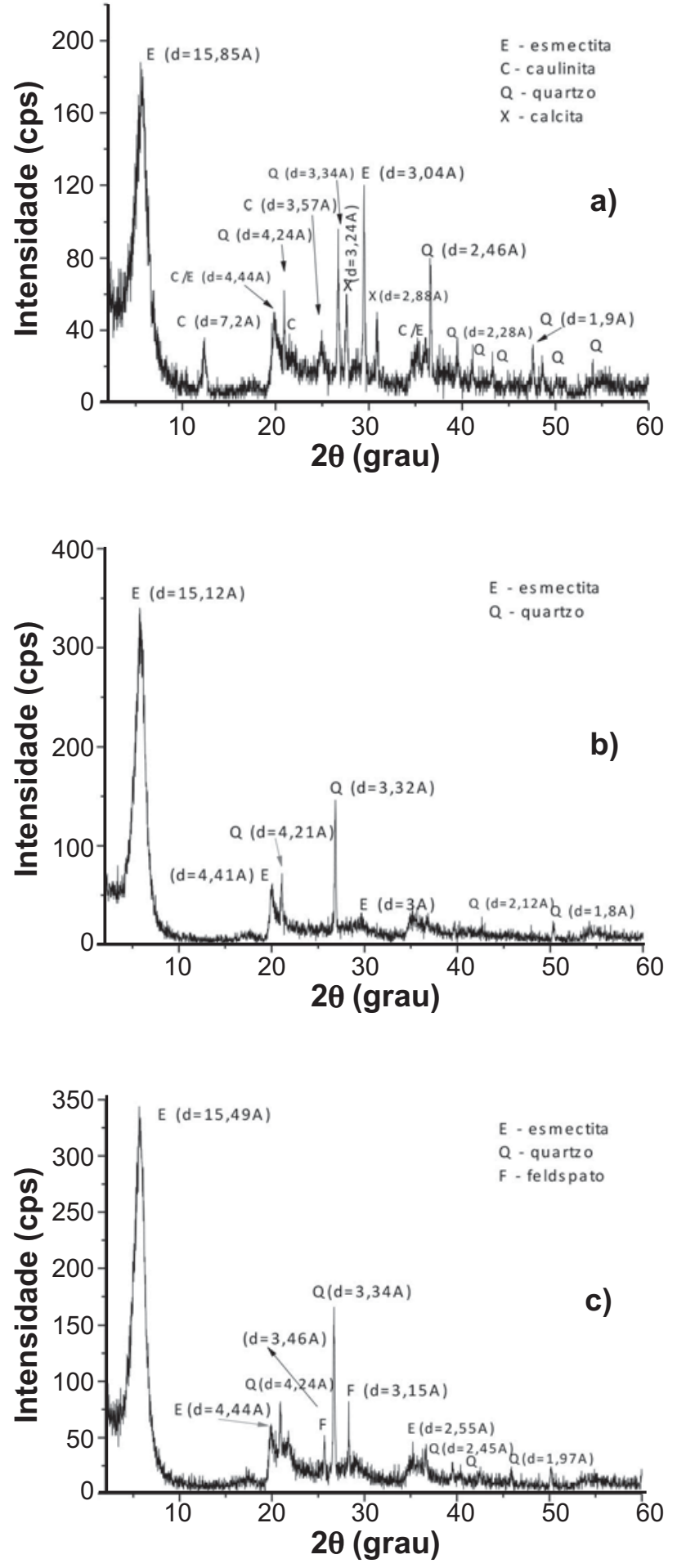

Figura 3: Difratogramas de raios $\mathrm{X}$ das amostras estudadas: (a) $\mathrm{CH} \mathrm{CN}$, (b) $\mathrm{CH} \mathrm{CO} \mathrm{e} \mathrm{(c)} \mathrm{CB} \mathrm{PR.}$

[Figure 3: X-ray diffraction patterns of studied samples: (a) $\mathrm{CHCN}$, (b) $\mathrm{CHCO}$ and (c) $\mathrm{CB}$ PR.]

$\mathrm{Na}_{2} \mathrm{CO}_{3}$ atingiu os valores normatizados para VA, VP e VF, segundo a norma da Petrobras [11]. No caso das amostras tratadas com $2 \%$ em massa de $\mathrm{Na}_{2} \mathrm{CO}_{3}$, a quantidade de defloculante é insuficiente, o que resulta em baixos valores 
Tabela II - Viscosidade aparente (VA), viscosidade plástica (VP) e volume de filtrado (VF) das amostras estudadas aditivadas com $2 \%$ e $3 \%$ em massa de $\mathrm{Na}_{2} \mathrm{CO}_{3}$.

[Table II - Apparent viscosity (VA), plastic viscosity (VP) and filtrate volume (VF) of the studied samples doped with $2 \%$ and $3 \%$ by weight of $\mathrm{Na}_{2} \mathrm{CO}_{3}$.]

\begin{tabular}{ccccccc}
\hline \multirow{2}{*}{ Amostra } & \multicolumn{2}{c}{$2 \%$ em massa de $\mathrm{Na}_{2} \mathrm{CO}_{3}$} & \multicolumn{4}{c}{$3 \%$ em massa de $\mathrm{Na}_{2} \mathrm{CO}_{3}$} \\
& $\mathrm{VA}(\mathrm{mPa} . \mathrm{s})$ & $\mathrm{VP}(\mathrm{mPa} . \mathrm{s})$ & $\mathrm{VF}(\mathrm{mL})$ & $\mathrm{VA}(\mathrm{mPa} . \mathrm{s})$ & $\mathrm{VP}(\mathrm{mPa} . \mathrm{s})$ & $\mathrm{VF}(\mathrm{mL})$ \\
\hline CH CN & 3,00 & 1,50 & 39,00 & 13,50 & 2,00 & 30,00 \\
CH CO & 9,75 & 4,00 & 20,00 & 19,00 & 5,00 & 17,20 \\
CB PR & 7,00 & 4,00 & 23,00 & 7,75 & 4,50 & 21,00 \\
\hline
\end{tabular}

de VA. As amostras tratadas com 3\% em massa de $\mathrm{Na}_{2} \mathrm{CO}_{3}$ resultam em aumentos de VA e VP e diminuição de VF, indicando um estado de defloculação mais adequado [16].

Na Tabela III estão contidos os resultados de VA, VP e VF das amostras aditivadas com $\mathrm{MgO}$ ( $2 \%$ e $3 \%$ em massa). Observa-se que as dispersões apresentaram VA em torno de 3,00 mPa.s, VP variando de 1,50 a 2,00 mPa.s e VF variando de 116,00 até $129,00 \mathrm{~mL}$ para a amostra $\mathrm{CH}$ CN. Observa-se VA variando entre 5,00 a 5,50 mPa.s, VP variando entre 2,00 a 3,00 mPa.s e VF variando entre 89,00 até $108,50 \mathrm{~mL}$ para a amostra $\mathrm{CH}$ CO. Observa-se também VA variando entre 2,50 a 3,00 mPa.s, VP variando entre 2,00 a 2,50 mPa.s e VF variando entre 116,00 até $128,00 \mathrm{~mL}$ para a amostra CB PR. Evidencia-se que nenhuma das dispersões aditivadas com $2 \%$ e $3 \%$ (em massa) de $\mathrm{MgO}$ satisfez a norma da Petrobras
[11]. As amostras tratadas com 2\% e 3\% em massa de $\mathrm{MgO}$, pelo fato do $\mathrm{Mg}(\mathrm{OH})_{2}$ ser um forte floculante, resultaram em baixos valores de VA e VP e elevados valores de VF, típicos de dispersões floculadas com separação de fase. Segundo [19], dispersões em $\mathrm{pH}$ 9,5 transformam o $\mathrm{Mg}^{2+}$ em $\mathrm{MgOH}^{+}$e $\mathrm{Mg}(\mathrm{OH})_{2}$, dependendo da concentração de $\mathrm{MgO}$, resultando em altas viscosidades pela estrutura do gel formado; contudo, quando o $\mathrm{Mg}(\mathrm{OH})_{2}$ é formado em $\mathrm{pHs}$ mais elevados, é precipitado juntamente com as partículas do argilomineral havendo heterocoagulação, destruindo a estrutura de castelo de cartas existente, resultando na separação de fases do sistema com redução da VA.

Nas Tabelas IV a VI estão contidos os resultados das propriedades reológicas obtidas a partir das amostras dispersas a 4,86\% (em massa) aditivadas com $\mathrm{Na}_{2} \mathrm{CO}_{3}$ em

Tabela III - Viscosidade aparente (VA), viscosidade plástica (VP) e volume de filtrado (VF) das amostras estudadas aditivadas com $2 \%$ e $3 \%$ em massa de $\mathrm{MgO}$.

[Table III - Apparent viscosity (VA), plastic viscosity (VP) and filtrate volume (VF) of the studied samples doped with $2 \%$ and $3 \%$ by weight of $\mathrm{MgO}$.]

\begin{tabular}{ccccccc}
\hline \multirow{2}{*}{ Amostra } & \multicolumn{2}{c}{$2 \%$ em massa de $\mathrm{MgO}$} & \multicolumn{5}{c}{$3 \%$ em massa de MgO } \\
& VA (mPa.s) & VP (mPa.s) & VF (mL) & VA (mPa.s) & VP (mPa.s) & VF (mL) \\
\hline CH CN & 3,00 & 2,00 & 116,00 & 3,00 & 1,50 & 129,00 \\
CH CO & 5,50 & 3,00 & 89,00 & 5,00 & 2,00 & 108,50 \\
CB PR & 2,50 & 2,50 & 116,00 & 3,00 & 2,00 & 128,00 \\
\hline
\end{tabular}

Tabela IV - Viscosidade aparente (VA), viscosidade plástica (VP) e volume de filtrado (VF) das amostras estudadas aditivadas com $2 \%$ e $3 \%$ em massa de $\mathrm{Na}_{2} \mathrm{CO}_{3}$ variando a concentração de $\mathrm{MgO}$ para a amostra $\mathrm{CH} \mathrm{CN}$.

[Table IV - Apparent viscosity (VA), plastic viscosity (PV) and filtrate volume (VF) of the studied samples doped with $2 \%$ and $3 \%$ by weight of $\mathrm{Na}_{2} \mathrm{CO}_{3}$ varying the concentration of $\mathrm{MgO}$ in the sample $\mathrm{CH} \mathrm{CN}$.]

\begin{tabular}{ccccccc}
\hline \multirow{2}{*}{ Conc. $\mathrm{MgO}(\%)$} & \multicolumn{2}{c}{ \% em massa de $\mathrm{Na}_{2} \mathrm{CO}_{3}$} & \multicolumn{3}{c}{$3 \%$ em massa de $\mathrm{Na}_{2} \mathrm{CO}_{3}$} \\
& $\mathrm{VA}(\mathrm{mPa} . \mathrm{s})$ & $\mathrm{VP}(\mathrm{mPa} . \mathrm{s})$ & $\mathrm{VF}(\mathrm{mL})$ & $\mathrm{VA}(\mathrm{mPa} . \mathrm{s})$ & $\mathrm{VP}(\mathrm{mPa} . \mathrm{s})$ & $\mathrm{VF}(\mathrm{mL})$ \\
\hline 0,5 & 12,50 & 3,00 & 29,00 & 15,00 & 4,00 & 34,00 \\
1,0 & 14,25 & 3,50 & 38,00 & 15,50 & 1,00 & 43,00 \\
1,5 & 11,00 & 5,00 & 49,00 & 12,0 & 4,50 & 49,00 \\
2,0 & 10,50 & 4,00 & 52,00 & 13,0 & 6,00 & 54,00 \\
3,0 & 5,25 & 3,00 & 77,00 & 7,75 & 4,50 & 70,00 \\
4,0 & 7,50 & 3,00 & 64,00 & 8,50 & 4,50 & 69,00 \\
\hline
\end{tabular}


conjunto com o $\mathrm{MgO}$ e sua comparação com a norma da Petrobras [11]. Na Tabela IV estão contidos os resultados de $\mathrm{VA}, \mathrm{VP}$ e VF da amostra $\mathrm{CH} \mathrm{CN}$ aditivada com $\mathrm{Na}_{2} \mathrm{CO}_{3}(2 \%$ e $3 \%$ em massa) combinados com a variação da concentração de $\mathrm{MgO}$. Observa-se que a bentonita $\mathrm{CH} \mathrm{CN}$ apresentou VA variando de 5,25 a 15,50 mPa.s, VP variando de 1,00 até 6,00 mPa.s e VF variando de 29,00 até $77 \mathrm{~mL}$. Comparando estes resultados com os das Tabelas II e III, pode-se observar uma melhora significativa nas propriedades reológicas quando do uso da aditivação conjunta para a amostra $\mathrm{CH}$ $\mathrm{CN}$. Observa-se que à medida que aumenta a concentração do $\mathrm{MgO}$ ocorre diminuição de VA e aumento do VF, pois dispersões tratadas com $\mathrm{Mg}(\mathrm{OH})_{2}$ quando em proporções mais elevadas apresentam comportamento de dispersões floculadas [19]. Sendo assim, ficou comprovada uma melhora nas propriedades reológicas em relação à adição individual, mas nenhuma dispersão analisada apresentou valores que atendessem a norma da Petrobras [11].

$\mathrm{Na}$ Tabela V estão contidos os resultados de VA, VP e $\mathrm{VF}$ da amostra $\mathrm{CH} \mathrm{CO}$ aditivada com $\mathrm{Na}_{2} \mathrm{CO}_{3}(2 \%$ e $3 \% \mathrm{em}$ massa) combinados com a variação da concentração de $\mathrm{MgO}$. Observa-se que a amostra estudada apresentou VA variando de 12,00 a 62,25 mPa.s, VP variando de 2,00 até 8,00 mPa.s e VF variando de 21,00 até 64,00 mL. Comparando estes resultados com os das Tabelas II e III, pode-se observar uma melhora significativa nas propriedades reológicas quando do uso da aditivação conjunta para a amostra CH CO. Observase aumento significativo das propriedades reológicas VA, VP e VF, o que evidencia um estado excessivamente floculado sem separação de fases, o que resultaria em valores de VA e VP muito baixos e VF muito elevado, o que não ocorreu, estando estes fenômenos de acordo com os dados reportados [16, 19]; contudo, nenhuma dispersão analisada apresentou valores que atendessem a norma da Petrobras [11].

$\mathrm{Na}$ Tabela VI estão contidos os resultados de VA, VP e VF da amostra CB PR aditivada com $\mathrm{Na}_{2} \mathrm{CO}_{3}(2 \%$ e $3 \%$ em massa) combinados com a variação da concentração de $\mathrm{MgO}$. Observa-se que a amostra apresentou VA variando de 3,75 a 14,50 mPa.s, VP variando de 2,00 até 5,00 $\mathrm{mPa} . \mathrm{s}$ e VF variando de 24,00 a 138,00 mL. Comparando estes resultados com os das Tabelas II e III, pode-se observar uma pequena melhora nas propriedades reológicas quando do uso da aditivação conjunta para a amostra CB PR. Observa-se que à medida que aumenta a concentração do $\mathrm{MgO}$ ocorre diminuição de VA e VP e aumento do VF, pois $\mathrm{o} \mathrm{Mg}(\mathrm{OH})_{2}$ formado precipita juntamente com as partículas do argilomineral havendo heterocoagulação, destruindo assim a estrutura de castelo de cartas existente, resultando na separação de fases do sistema, com redução da VA e VP e consequentemente aumento de VF [19]. Desta forma,

Tabela V - Viscosidade aparente (VA), viscosidade plástica (VP) e volume de filtrado (VF) das amostras estudadas aditivadas com $2 \%$ e $3 \%$ em massa de $\mathrm{Na}_{2} \mathrm{CO}_{3}$ variando a concentração de $\mathrm{MgO}$ para a amostra $\mathrm{CH} \mathrm{CO}$.

[Table V-Apparent viscosity (VA), plastic viscosity (VP) and filtrate volume (VF) of the studied samples doped with $2 \%$ and $3 \%$ by weight of $\mathrm{Na}_{2} \mathrm{CO}_{3}$ varying the concentration of $\mathrm{MgO}$ in the sample $\left.\mathrm{CH} \mathrm{CO}.\right]$

\begin{tabular}{ccccccc}
\hline \multirow{2}{*}{ Conc. $\mathrm{MgO}(\%)$} & \multicolumn{2}{c}{$2 \%$ em massa de $\mathrm{Na}_{2} \mathrm{CO}_{3}$} & \multicolumn{4}{c}{$3 \%$ em massa de $\mathrm{Na}_{2} \mathrm{CO}_{3}$} \\
& $\mathrm{VA}(\mathrm{mPa} . \mathrm{s})$ & $\mathrm{VP}(\mathrm{mPa} . \mathrm{s})$ & $\mathrm{VF}(\mathrm{mL})$ & $\mathrm{VA}(\mathrm{mPa} . \mathrm{s})$ & $\mathrm{VP}(\mathrm{mPa} . \mathrm{s})$ & $\mathrm{VF}(\mathrm{mL})$ \\
\hline 0,5 & 14,50 & 3,50 & 21,00 & 62,25 & 4,00 & 22,00 \\
1,0 & 29,00 & 2,00 & 25,00 & 61,00 & 3,00 & 26,00 \\
1,5 & 28,00 & 6,00 & 34,00 & 33,75 & 7,00 & 34,00 \\
2,0 & 16,50 & 3,50 & 44,00 & 27,50 & 8,00 & 42,00 \\
3,0 & 24,25 & 4,50 & 45,00 & 22,50 & 6,00 & 48,00 \\
4,0 & 12,00 & 5,00 & 61,00 & 12,00 & 5,50 & 64,00 \\
\hline
\end{tabular}

Tabela VI - Viscosidade aparente (VA), viscosidade plástica (VP) e volume de filtrado (VF) das amostras estudadas aditivadas com $2 \%$ e $3 \%$ em massa de $\mathrm{Na}_{2} \mathrm{CO}_{3}$ variando a concentração de $\mathrm{MgO}$ para a amostra CB PR. [Table VI - Apparent viscosity (VA), plastic viscosity (VP) and filtrate volume (VF) of the studied samples doped with $2 \%$ and $3 \%$ by weight of $\mathrm{Na}_{2} \mathrm{CO}_{3}$ varying the concentration of $\mathrm{MgO}$ in the sample CB PR.]

\begin{tabular}{ccccccc}
\hline \multirow{2}{*}{ Conc. $\mathrm{MgO}(\%)$} & \multicolumn{2}{c}{$3 \%$ em massa de $\mathrm{Na}_{2} \mathrm{CO}_{3}$} & \multicolumn{3}{c}{$3 \% \mathrm{Na}_{2} \mathrm{CO}_{3}$} \\
& $\mathrm{VA}(\mathrm{mPa} . \mathrm{s})$ & $\mathrm{VP}(\mathrm{mPa} . \mathrm{s})$ & $\mathrm{VF}(\mathrm{mL})$ & $\mathrm{VA}(\mathrm{mPa} . \mathrm{s})$ & $\mathrm{VP}(\mathrm{mPa} . \mathrm{s})$ & $\mathrm{VF}(\mathrm{mL})$ \\
\hline 0,5 & 7,75 & 3,50 & 24,00 & 14,50 & 4,00 & 27,00 \\
1,0 & 4,75 & 2,50 & 34,00 & 7,00 & 4,00 & 68,00 \\
1,5 & 5,50 & 3,00 & 34,00 & 10,00 & 5,00 & 54,00 \\
2,0 & 5,75 & 2,50 & 40,00 & 6,50 & 4,00 & 76,00 \\
3,0 & 6,00 & 3,00 & 54,00 & 5,75 & 4,00 & 93,00 \\
4,0 & 3,75 & 3,00 & 106,00 & 3,75 & 2,00 & 138,00 \\
\hline
\end{tabular}


nenhuma das dispersões analisadas apresentou valores que atendessem a norma da Petrobras [11].

Nas Tabelas VII e VIII estão contidos os resultados das propriedades reológicas obtidas a partir das amostras dispersas a $6,00 \%$ (em massa) e aditivadas isoladamente. Na Tabela VII estão contidos os resultados de VA e VF das amostras estudadas aditivadas com $\mathrm{Na}_{2} \mathrm{CO}_{3}(2 \%$ e $3 \%$ em massa). Observa-se que as dispersões apresentaram VA variando de 3,00 a 13,50 mPa.s e VF variando de 30,00 até 39,00 mL para a amostra CH CN. Observa-se VA variando entre 9,75 a 19,00 mPa.s e VF variando entre 17,20 até 20,00 $\mathrm{mL}$ para a amostra $\mathrm{CH}$ CO. Observa-se também VA variando entre 7,00 a 7,75 mPa.s e VF variando entre 21,00 até 23,00 $\mathrm{mL}$ para a amostra CB PR. No caso das amostras tratadas com $2 \%$ em massa de $\mathrm{Na}_{2} \mathrm{CO}_{3}$, a quantidade de defloculante é insuficiente o que resulta em baixos valores de VA. As amostras tratadas com $3 \%$ em massa de $\mathrm{Na}_{2} \mathrm{CO}_{3}$ resultam em aumentos de VA e VP e diminuição de VF, indicando um estado de defloculação mais adequado [16]; nenhuma das amostras, entretanto, satisfazem os valores normatizados para VA e VF segundo as normas da API [12] e ISO [13].

Na Tabela VIII estão contidos os resultados de VA e VF das amostras estudadas aditivadas com $\mathrm{MgO}(2 \%$ e $3 \%$ em massa). Observa-se que as dispersões apresentaram VA em torno de 3,00 mPa.s e VF variando de 116,00 até 129,00 mL

Tabela VII - Viscosidade aparente (VA) e volume de filtrado (VF) das amostras estudadas aditivadas com $2 \%$ e $3 \%$ em massa de $\mathrm{Na}_{2} \mathrm{CO}_{3}$.

[Table VII - Apparent viscosity (VA) and filtrate volume (VF) of the studied samples doped with $2 \%$ and $3 \%$ by weight of $\mathrm{Na}_{2} \mathrm{CO}_{3}$. $]$

\begin{tabular}{ccccc}
\hline & $\begin{array}{c}2 \% \text { em massa de } \\
\mathrm{Na}_{2} \mathrm{CO}_{3}\end{array}$ & \multicolumn{2}{c}{$\begin{array}{c}3 \% \text { em massa de } \\
\mathrm{Na}_{2} \mathrm{CO}_{3}\end{array}$} \\
& $\mathrm{VA}(\mathrm{mPa} . \mathrm{s})$. & $\mathrm{VF}(\mathrm{mL})$ & $\mathrm{VA}(\mathrm{mPa} . \mathrm{s})$. & $\mathrm{VF}(\mathrm{mL})$ \\
\hline CH CN & 3,00 & 39,00 & 13,50 & 30,00 \\
CH CO & 9,75 & 20,00 & 19,00 & 17,20 \\
$\mathrm{CB} \mathrm{PR}$ & 7,00 & 23,00 & 7,75 & 21,00 \\
\hline
\end{tabular}

Tabela VIII - Viscosidade aparente (VA) e volume de filtrado (VF) das amostras estudadas aditivadas com $2 \%$ e $3 \%$ em massa de $\mathrm{MgO}$.

[Table VIII - Apparent viscosity (VA) and filtrate volume (VF) of the studied samples doped with $2 \%$ and $3 \%$ by weight of $\mathrm{MgO}$.]

\begin{tabular}{ccccc}
\hline \multirow{2}{*}{ Amostra } & \multicolumn{2}{c}{$2 \%$ em massa de $\mathrm{MgO}$} & \multicolumn{2}{c}{$3 \%$ em massa de $\mathrm{MgO}$} \\
& $\mathrm{VA}(\mathrm{mPa} . \mathrm{s})$ & $\mathrm{VF}(\mathrm{mL})$ & $\mathrm{VA}(\mathrm{mPa} . \mathrm{s})$ & $\mathrm{VF}(\mathrm{mL})$ \\
\hline $\mathrm{CH} \mathrm{CN}$ & 3,00 & 116,00 & 3,00 & 129,00 \\
$\mathrm{CH} \mathrm{CO}$ & 5,50 & 89,00 & 5,00 & 108,50 \\
$\mathrm{CB}$ PR & 2,50 & 116,00 & 3,00 & 128,00 \\
\hline
\end{tabular}

para a amostra $\mathrm{CH}$ CN. Observa-se VA variando entre 5,00 e 5,50 mPa.s e VF variando entre 89,00 até 108,50 mL para a amostra $\mathrm{CH} \mathrm{CO}$. Observa-se também VA variando entre 2,50 e 3,00 mPa.s e VF variando entre 116,00 até 128,00 $\mathrm{mL}$ para a amostra CB PR. Evidencia-se que nenhuma das dispersões aditivadas com $2 \%$ e $3 \%$ (em massa) de $\mathrm{MgO}$ satisfez os limites máximos especificados pelas normas da API [12] e ISO [13]. Os fenômenos envolvidos são idênticos aos já descritos quando da análise da Tabela III.

Nas Tabelas IX a XI estão contidos os resultados das propriedades reológicas obtidas a partir das amostras dispersas a 6,00\% (em massa) aditivadas com $\mathrm{Na}_{2} \mathrm{CO}_{3} \mathrm{em}$ conjunto com o $\mathrm{MgO}$ e sua comparação com as normas da API [12] e ISO [13]. Na Tabela IX estão contidos os resultados de VA e VF da amostra $\mathrm{CH} \mathrm{CN}$ aditivada com $\mathrm{Na}_{2} \mathrm{CO}_{3}(2 \%$ e $3 \%$ em massa) combinada com a variação da concentração de $\mathrm{MgO}$. Observa-se que a bentonita $\mathrm{CH} \mathrm{CN}$ apresentou VA variando de 17,00 a 37,50 mPa.s e VF variando de 27,00 até 49,50 mL. Comparando estes resultados com os das Tabelas VII e VIII, pode-se observar uma melhora significativa nas propriedades reológicas quando do uso da aditivação conjunta para a amostra $\mathrm{CH}$ CN. Os fenômenos envolvidos são idênticos aos já descritos quando da análise da Tabela IV. Sendo assim, ficou comprovada uma melhora nas propriedades reológicas em relação à adição individual, mas nenhuma das dispersões analisadas apresentou valores que atendessem as especificações das normas da API [12] e ISO [13].

Na Tabela X estão contidos os resultados de VA e VF da amostra $\mathrm{CH} \mathrm{CO}$ aditivada com $\mathrm{Na}_{2} \mathrm{CO}_{3}$ (2\% e $3 \%$ em massa) combinados com a variação da concentração de $\mathrm{MgO}$. Observa-se que a amostra estudada apresentou VA variando de 28,00 a 111,50 mPa.s e VF variando de 16,00 até 44,00 $\mathrm{mL}$. Comparando estes resultados com os das Tabelas VII e VIII, pode-se observar uma melhora significativa nas propriedades reológicas quando da aditivação conjunta para

Tabela IX - Viscosidade aparente (VA) e volume de filtrado (VF) das amostras estudadas aditivadas com $2 \%$ e $3 \%$ em massa de $\mathrm{Na}_{2} \mathrm{CO}_{3}$ variando a concentração de $\mathrm{MgO}$ para a amostra $\mathrm{CH} \mathrm{CN}$.

[Table IX - Apparent viscosity (VA) and filtrate volume (VF) of the studied samples doped with $2 \%$ and $3 \%$ by weight of $\mathrm{Na}_{2} \mathrm{CO}_{3}$ varying the concentration of $\mathrm{MgO}$ in the sample $\mathrm{CH} \mathrm{CN}$.]

\begin{tabular}{ccccc}
\hline & \multicolumn{2}{c}{$\begin{array}{c}2 \% \text { em massa } \\
\text { Conc. } \\
\mathrm{MgO}(\%)\end{array}$} & $\begin{array}{c}3 \% \text { em massa } \\
\text { de } \mathrm{Na}_{2} \mathrm{CO}_{3}\end{array}$ & \multicolumn{2}{c}{$\begin{array}{c}\text { de } \mathrm{CO}_{3} \\
\text { VAa.s) }\end{array}$} & $\mathrm{VF}(\mathrm{mL})$ & $\mathrm{VA}(\mathrm{mPa} . \mathrm{s})$ & $\mathrm{VF}(\mathrm{mL})$ \\
\hline 0,5 & 19,50 & 28,00 & 33,75 & 27,00 \\
1,0 & 21,75 & 32,00 & 37,50 & 32,00 \\
1,5 & 22,50 & 25,00 & 34,00 & 35,00 \\
2,0 & 18,50 & 43,00 & 27,00 & 40,00 \\
3,0 & 17,50 & 47,00 & 23,50 & 47,00 \\
4,0 & 17,00 & 49,00 & 22,50 & 49,50 \\
\hline
\end{tabular}


Tabela X - Viscosidade aparente (VA) e volume de filtrado (VF) das amostras estudadas aditivadas com $2 \%$ e $3 \%$ em massa de $\mathrm{Na}_{2} \mathrm{CO}_{3}$ variando a concentração de $\mathrm{MgO}$ para a amostra $\mathrm{CH} \mathrm{CO}$.

[Table X-Apparent viscosity (AV) and filtrate volume (VF) of the studied samples doped with $2 \%$ and $3 \%$ by weight of $\mathrm{Na}_{2} \mathrm{CO}_{3}$ varying the concentration of $\mathrm{MgO}$ in the sample $\mathrm{CHCO}$.]

\begin{tabular}{ccccc}
\hline & \multicolumn{2}{c}{$\begin{array}{c}2 \% \text { em massa de } \\
\mathrm{Na}_{2} \mathrm{CO}_{3}\end{array}$} & \multicolumn{3}{c}{$\begin{array}{c}\text { 3\% em massa de } \\
\mathrm{Na}_{2} \mathrm{CO}_{3}\end{array}$} \\
\hline$\%)$ & $\mathrm{VA}(\mathrm{mPa} . \mathrm{s})$ & $\mathrm{VF}(\mathrm{mL})$ & $\mathrm{VA}(\mathrm{mPa} . \mathrm{s})$ & $\mathrm{VF}(\mathrm{mL})$ \\
\hline 0,5 & 28,00 & 15,00 & 95,50 & 18,10 \\
1,0 & 39,00 & 22,00 & 111,50 & 21,50 \\
1,5 & 39,50 & 29,00 & 76,50 & 27,50 \\
2,0 & 50,50 & 32,00 & 58,00 & 33,50 \\
3,0 & 26,00 & 41,00 & 55,50 & 38,00 \\
4,0 & 25,50 & 44,00 & 37,50 & 44,00 \\
\hline
\end{tabular}

Tabela XI - Viscosidade aparente (VA) e volume de filtrado (VF) das amostras estudadas aditivadas com $2 \%$ e $3 \%$ em massa de $\mathrm{Na}_{2} \mathrm{CO}_{3}$ variando a concentração de $\mathrm{MgO}$ para a amostra $\mathrm{CB} \mathrm{PR}$.

[Table XI - Apparent viscosity (AV), plastic viscosity (PV) and filtrate volume (VF) of the studied samples doped with $2 \%$ and $3 \%$ by weight of $\mathrm{Na}_{2} \mathrm{CO}_{3}$ varying the concentration of $\mathrm{MgO}$ in the sample $\mathrm{CB} P R$.]

\begin{tabular}{ccccc}
\hline \multirow{2}{*}{$\begin{array}{c}\text { Conc. } \mathrm{MgO} \\
(\%)\end{array}$} & \multicolumn{2}{c}{$\begin{array}{c}2 \% \text { em massa de } \\
\mathrm{Na}_{2} \mathrm{CO}_{3}\end{array}$} & \multicolumn{2}{c}{$3 \%$ em massa de } \\
$\mathrm{Na}_{2} \mathrm{CO}_{3}$
\end{tabular}

a amostra CH CO. Os fenômenos envolvidos são idênticos aos já descritos quando da análise da Tabela V; apenas a amostra aditivada com $2 \%$ em massa de $\mathrm{Na}_{2} \mathrm{CO}_{3}+0,5 \%$ em massa de $\mathrm{MgO}$ apresentou valores que atenderam as especificações das normas da API [12] e ISO [13].

Na Tabela XI estão contidos os resultados de VA e VF da amostra CB PR aditivada com $\mathrm{Na}_{2} \mathrm{CO}_{3}$ (2\% e $3 \%$ em massa) combinada com a variação da concentração de $\mathrm{MgO}$. Podese notar que a amostra estudada apresentou VA variando de 6,25 a 40,00 mPa.s e VF variando de 20,00 até 96,50 $\mathrm{mL}$. Comparando estes resultados com os das Tabelas VII e VIII, pode-se observar uma melhora significativa nas propriedades reológicas quando da aditivação conjunta para a amostra CB PR. Os fenômenos envolvidos são idênticos aos já descritos quando da análise da Tabela VI; contudo, nenhuma das dispersões analisadas apresentou valores que atendessem as especificações das normas da API [12] e ISO [13].

\section{CONCLUSÕES}

Após a análise dos dados obtidos, pode-se concluir que todas as amostras estudadas apresentaram composições mineralógicas típicas das bentonitas oriundas do estado da Paraíba, apresentando os argilominerais característicos esmectíticos, cauliníticos e acessórios. Dentre as aditivações realizadas isoladamente, apenas a amostra $\mathrm{CH}$ CO dispersa a uma concentração de 4,86\% em massa e aditivada com $3 \%$ (em massa) de $\mathrm{Na}_{2} \mathrm{CO}_{3}$ atingiu os valores especificados pela norma da Petrobras [11] para fluidos de perfuração. Dentre as aditivações conjuntas estudadas, observou-se que apenas a amostra $\mathrm{CH} \mathrm{CO}$ na proporção de $2 \%$ (em massa) de $\mathrm{Na}_{2} \mathrm{CO}_{3}$ $+0,5 \%$ (em massa) de $\mathrm{MgO}$ atingiu as especificações das normas da API [12] e ISO [13] para tintas à base de água.

\section{REFERÊNCIAS}

[1] P.S. Santos, Ciência e Tecnologia de Argilas, 2a Ed., Vols. 1, 2 e 3, Edgard Blücher, S. Paulo (1992).

[2] A.R.V. Silva, H. C. Ferreira, "Argilas bentoníticas: conceitos, estruturas, propriedades, síntese, usos industriais e produtores/fornecedores nacionais e internacionais", Revista Eletrônica Materiais Processos (REMAP) 3, 2 (2008) 26-35.

[3] I.D.S. Pereira, V.N.F. Lisboa, I.A. Silva, J.M.R. Figueirêdo, G.A. Neves, R.R. Menezes, "Bentonite clays from Sossego, Paraíba, Brazil: physical and mineralogical characterization", Mater. Sci. Forum 798-799 (2014) 50-54. [4] H.H. Murray, Applied Clay Mineralogy - Occurrences, processing and applications of kaolin, bentonites, palygorskite-sepiolite, and common clays, Developments in Clay Science, Vol. 2, Elsevier, Oxford, UK (2006).

[5] DNPM, Departamento Nacional de Produção Mineral. Anuário Mineral Brasileiro, Bentonita (2009).

[6] R. Barbosa, D.D.S. Morais, E.M. Araujo, T.J.A. Melo, "Caracterizações de argilas bentoníticas e síntese de argilas organofílicas para uso em nanocompósitos poliméricos: parte II", Cerâmica 58, 347 (2012) 363-367.

[7] R.C.A.M. Nascimento, A.C.A. Costa, L.V. Amorim, "Desempenho de fluidos argilosos envelhecidos termicamente", Revista Eletrônica de Materiais e Processos (REMAP) 7, 3 (2012) 205-214.

[8] R.C.A.M. Nascimento, L.V. Amorim, L.N.L. Santana, "Desenvolvimento de fluidos aquosos com bentonita para perfuração de poços de petróleo on shore", Cerâmica 56, 338 (2010) 179-178.

[9] J.E. Thomas, Fundamentos de Engenharia de Petróleo, Interciência, Rio de Janeiro, RJ (2001).

[10] X. Meng, Y. Zhang, F. Zhou, Chu, K. Paul, "Effects of carbon ash on rheological properties of water-based drilling fluids", J. Pet. Sci. Eng. 100 (2012) 1-8.

[11] Petrobras, Ensaio de viscosificante para fluidos base água na exploração e produção de petróleo, EP-1EP- 
00011-A (2011).

[12] American Petroleum Institute, Recommended practice for field testing water-based drilling fluids, API 13B-1 (2003).

[13] ISO, Petroleum and natural gas industries - Drilling fluids materials - Specifications and tests, ISO 13500 (2008). [14] J.C.V. Machado, Reologia de fluidos: aplicação na engenharia de petróleo, Centro de Pesquisa e Desenvolvimento da Petrobras, Salvador, BA (1983).

[15] J.C.V. Machado, Reologia e escoamento de fluidos, Interciência, Rio de Janeiro (2002).

[16] A.N. Van Olphen, Introduction to Clay Colloid Chemistry, $2^{\text {a }}$ Ed., John Wiley \& Sons, New York (1977).

[17] M.I. Barbosa, L.V. Amorim, H.C. Ferreira, "Compostos poliméricos como aditivos de argilas bentoníticas", Cerâmica 53, 328 (2007) 354-360.

[18] F. Karakas, G. Pyrgiotakis, M.S. Çelik, B.M. Moudgil, "Na-bentonite and $\mathrm{MgO}$ mixture as a thickening agent for water-based paints", KONA Powder Part. J. 29 (2011) 96106.
[19] C. Karagüzel, A.T. Çetinel, F. Boylub, K. Çinkuc, M.S. Çelik, "Activation of ( $\mathrm{Na}, \mathrm{Ca})$-bentonites with soda and $\mathrm{MgO}$ and their utilizationas drilling mud", Appl. Clay Sci. 48 (2010) 398-404.

[20] J. Abdo, A. Al-Shabibi, H. Al-Sharji, "Effects of tribological properties of water-based drilling fluids on buckling and lock-up length of coiled tubing in drilling operations", Tribology Int. 82 (2014) 493-503.

[21] R.R. Menezes, P.M. Souto, L.N.L. Santana, G.A. R. Neves, H.G.A. Kiminami, H.C. Ferreira, "Argilas bentoníticas de Cubati, Paraíba, Brasil: Caracterização física-mineralógica", Cerâmica 55, 334 (2009) 163-169.

[22] I.A. Silva, J.M.R. Costa, R.R. Menezes, H.S. Ferreira, G.A. Neves, H.C. Ferreira, "Studies of new occurrences of bentonite clays in the State of Paraíba for use in water based drilling fluids", Esc. Minas (REM) 66, 4 (2013) 485-491.

[23] P.S. Santos, Tecnologia de Argilas, $2^{\mathrm{a}}$ Ed., Vol. 1, Edgard Blücher, S. Paulo (1989).

(Rec. 02/09/2015, Rev. 02/10/2015, 08/10/2015, Ac. $14 / 10 / 2015$ 